\title{
EI virus del mosaico amarillo de la papa de Panamá (PYMPV): un patógeno emergente que infecta tomate industrial en la provincia de Los Santos, Panamá
}

\author{
Potato yellow mosaic Panama virus (PYMPV): an emerging pathogen \\ infecting industrial tomato in Los Santos province, Panama \\ José Ángel Herrera-Vásquez ${ }^{*}$, José Natividad Jaén-Sanjur², \\ Anovel Amet Barba Alvarado ${ }^{1}$, Vidal Antonio Aguilera Cogley ${ }^{1}$
}

\section{RESUMEN}

Los begomovirus (género Begomovirus, familia Geminiviridae) constituyen el principal problema fitosanitario del cultivo de tomate en las regiones tropicales y subtropicales del mundo. Estos virus se transmiten por la mosca blanca Bemisia tabaci, la que en los últimos años ha tenido un incremento significativo en sus poblaciones, y se ha asociado con la aparición de síntomas virales en cultivos de tomate en Panamá. Se realizó una prospección en cultivos de tomate industrial en la provincia de Los Santos en el período 2016-2017, con el objetivo de determinar la incidencia de begomovirus en esta región. Se recolectaron 70 muestras de hojas de plantas de tomate mostrando síntomas asociados a infecciones virales, en nueve parcelas ubicadas en siete localidades distintas, quienes constituyen las principales zonas productoras de tomate en esta provincia. Se realizó la extracción de ADN de cada muestra y los extractos se analizaron mediante la técnica de reacción en cadena de la polimerasa (PCR), utilizando dos parejas de iniciadores degenerados para detectar begomovirus. Las muestras que mostraron una reacción positiva se analizaron con iniciadores específicos para identificar las tres especies de begomovirus que infectan tomate en Panamá, siendo estas, el virus del mosaico amarillo de la papa de Panamá (PYMPV), virus del moteado amarillo del tomate (TYMoV) y el virus del enrollamiento de la hoja de tomate de Sinaloa (ToLCSiV). Cincuenta y ocho (58) muestras de tomate (82,9\% del total de muestras recolectadas) resultaron positivas a begomovirus. El 100\% de estas muestras resultó infectado con PYMPV. No se determinó la presencia de TYMoV y ToLCSiV en las muestras analizadas. Estos resultados se confirmaron mediante secuenciación de ADN y análisis de secuencias. Se discute en este estudio la asociación de PYMPV con los graves daños que ha sufrido recientemente el sector tomatero con la baja de su producción.

Palabras clave: begomovirus, reacción en cadena de la polimerasa, Solanum lycopersicum.

\begin{abstract}
Begomoviruses (genus Begomovirus, family Geminiviridae) constitute the main phytosanitary problem of tomato crop in tropical and subtropical regions of the world. These viruses are transmitted by the whitefly Bemisia tabaci, which in recent years has had a significant increase in its populations, and has been associated with the appearance of viral symptoms in tomato crops in Panama. A survey was carried out on industrial tomato crops from Los Santos province in the period 2016-2017, with the objective of determine the incidence of begomovirus in this region. Seventy leaf samples from tomato plants showing symptoms associated to viral infections were collected in nine plots located in seven distinct locations, which constitute the main tomato producing zones in this province. DNA extraction was performed from each sample and the extracts were analyzed by the polymerase chain reaction (PCR) technique, using two pairs of degenerate primers to detect the genus Begomovirus. Samples that showed a positive reaction were analyzed with specific primers to identify the three begomovirus species that infect tomato in Panama, being these, Potato yellow mosaic Panama virus (PYMPV), Tomato yellow mottle virus (TYMoV) and Tomato leaf curl Sinaloa virus (ToLCSiV). Fifty-eight (58) tomato samples (82.9\% of the total samples collected) were begomovirus positive. One hundred percent (100\%) of these samples was infected with PYMPV. The presence of TYMoV and ToLCSiV was not determined in the analyzed samples. These results were confirmed by DNA sequencing and sequences analysis. The association of PYMPV with the serious damage recently suffered by the tomato industry with the decrease of its production is discussed in this study.

Key words: begomovirus, polymerase chain reaction, Solanum lycopersicum.

1 Grupo de Investigación de Protección Vegetal (GIPV), Centro de Investigación Agropecuaria Central (CIAC), Instituto de Investigación Agropecuaria de Panamá (IDIAP), Herrera, Panamá.

2 Escuela de Biología, Facultad de Ciencias Naturales, Exactas y Tecnología (FCNET), Universidad de Panamá (UP), 3366 Panamá 4, Panamá.

* Autor para correspondencia: joshervs11@gmail.com
\end{abstract}

Fecha de Recepción: 19 Septiembre, 2017.

Fecha de Aceptación: 30 Octubre, 2017. 


\section{Introducción}

El tomate (Solanum lycopersicum L.) es la hortaliza más cultivada y de mayor valor económico en el mundo (Hanssen et al., 2010). Panamá ocupa el vigésimo segundo y el quinto lugar en producción de tomate en el continente americano y en América Central, respectivamente (FAO, 2017). En Panamá se cultiva, principalmente, tomate industrial, ocupando la provincia de Los Santos, en la región central del país, el primer lugar en producción nacional con 14.635 t $(84,1 \%)$ (INEC, 2017).

En la provincia de Los Santos se han observado plantas de tomate mostrando síntomas asociados a begomovirus desde 1983, pero el virus del mosaico amarillo de la papa de Panamá (PYMPV; género Begomovirus, familia Geminiviridae) se presentó como un problema a partir de 1991, con el incremento de las poblaciones de la mosca blanca Bemisia tabaci Gennadius (Hemiptera: Aleyrodidae), debido al uso indiscriminado de insecticidas (Engel et al., 1998). Recientemente, se reportó en Panamá la presencia de otras dos especies de begomovirus, el virus del moteado amarillo del tomate (TYMoV) y el virus del enrollamiento de la hoja de tomate de Sinaloa (ToLCSiV), afectando al tomate en las provincias de Herrera (región central) y Chiriquí (región occidental), respectivamente (Herrera-Vásquez et al., 2015, 2016). Los begomovirus presentan uno (monopartito) o dos (bipartito) componentes genómicos, denominados ADN-A y ADN-B, y se transmiten de forma persistente por B. tabaci (Jones, 2003). Las especies de begomovirus identificadas en Panamá poseen un genoma bipartito (Engel et al., 1998; Herrera-Vásquez et al., 2015, 2016).

En los años 1990, el promedio anual de la producción de tomate industrial en Panamá era de $36 \mathrm{t} / \mathrm{ha}$, con pérdidas de rendimiento de 2,2 t/ha por año. Esta situación condujo a pérdidas económicas para los productores de US\$220/ha por año, lo que equivalió a US\$1.000.000 durante el período 1991-1997, debido principalmente a las enfermedades causadas por PYMPV (Engel et al., 1998). De igual forma, en el período 2011-2012, la incidencia de este virus alcanzó proporciones epidémicas en la provincia de Los Santos y en otras regiones dedicadas al cultivo de tomate en Panamá (Herrera-Vásquez et al., 2016). Asimismo, en el período 2015-2016, se presentó una disminución de $6.000 \mathrm{t}(50 \%)$ de la producción de tomate industrial en la provincia de Los Santos, según informaron al periódico El Siglo de Panamá, la asociación de productores de tomate industrial de la provincia de Los Santos y el Ministerio de Desarrollo Agropecuario de Panamá (MIDA) (Mendoza, 2015).

La identificación de begomovirus debe ser un proceso permanente, dado que los nuevos patógenos requieren cambios continuos en las estrategias de manejo. Este estudio se realizó con el objetivo de determinar la incidencia actual de PYMPV, y su asociación con la reducción de la producción de tomate industrial informada recientemente en la provincia de Los Santos.

\section{Materiales y Métodos}

Se recolectaron 70 muestras de tomate industrial de diferentes cultivares locales ( $\mathrm{T}-7$, T-8 y criollo), cultivados a campo abierto en nueve parcelas ubicadas en siete localidades distintas de la provincia de Los Santos, Panamá, durante el período 2016-2017 (Figura 1, Tabla 1). Cada parcela se georreferenció con la ayuda de un sistema de posicionamiento global (GPS). En total, se tomaron muestras de plantas que mostraban síntomas asociados a begomovirus, con diferentes grados de severidad, incluyendo mosaico amarillo y enrollamiento de las hojas, reducción del tamaño de las plantas, maduración irregular y reducción del tamaño de los frutos (Figura 2). Se utilizó agua ultrapura estéril y se cultivaron plantas de tomate (cultivares T-7, T-8 y T-9) en una jaula a prueba de insectos, para ser utilizadas como controles negativos en la detección e identificación de begomovirus mediante la reacción en cadena de la polimerasa (PCR).

La extracción de ADN total de cada muestra se realizó siguiendo el protocolo indicado por Dellaporta et al. (1983). La detección de begomovirus se realizó mediante la técnica de PCR, siguiendo el procedimiento descrito por HerreraVásquez et al. (2016), utilizando dos parejas de iniciadores degenerados, PAL1v1978/PARc715 y PBL1v2040/PCRc2, los que amplifican parcialmente el ADN-A y el ADN-B de este grupo de virus, respectivamente (Rojas et al., 1993). La primera pareja de iniciadores amplifica un fragmento de $\sim 1.400$-pb, correspondiente a un segmento de la proteína asociada con la replicación (Rep), la 


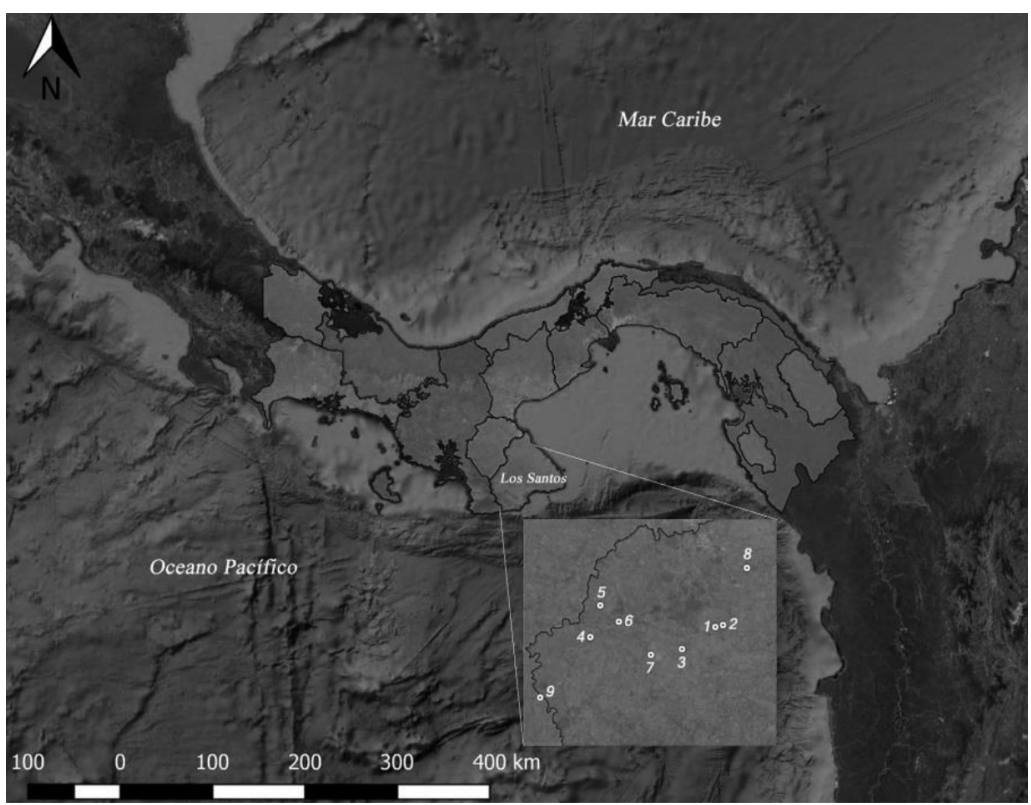

Figura 1. Localización geográfica del área muestreada en la provincia de Los Santos, Panamá (período 2016-2017): Nos 1 y 2 (Parcelas 1 y 2, Tres Quebradas), № 3 (Parcela 3, Las Cruces), $\mathrm{N}^{\circ} 4$ (Parcela 4, La Colorada), Nos 5 y 6 (Parcelas 5 y 6, San Luis), № 7 (Parcela 7, Villa Lourdes), № 8 (Parcela 8, El Ejido), y No 9 (Parcela 9, Chupá).

Tabla 1. Incidencia y distribución del virus del mosaico amarillo de la papa de Panamá (PYMPV) mediante PCR en muestras de tomate procedentes de diferentes localidades de la provincia de Los Santos, Panamá (período 2016-2017).

\begin{tabular}{|c|c|c|c|c|c|c|c|}
\hline \multirow[t]{2}{*}{ Localidad } & \multicolumn{2}{|c|}{$\frac{\text { GPS }}{\text { Coordenadas UTM }}$} & \multirow[t]{2}{*}{ Altituda } & \multirow[t]{2}{*}{ Muestras } & \multicolumn{2}{|c|}{$\begin{array}{c}\text { Begomovirus (bipartito), } \\
\text { Nuevo Mundo }\end{array}$} & \multirow{2}{*}{$\begin{array}{c}\begin{array}{c}\text { Especie de } \\
\text { begomovirus }^{\mathrm{b}}\end{array} \\
\text { PYMPV }\end{array}$} \\
\hline & $\mathrm{X}$ & Y & & & ADN-A & ADN-B & \\
\hline Parcela 1: Tres Quebradas & 566823 & 867500 & 64 & 5 & 5 & 5 & 5 \\
\hline Parcela 2: Tres Quebradas & 565962 & 867286 & 72 & 10 & 9 & 9 & 9 \\
\hline Parcela 3: Las Cruces & 562230 & 864838 & 83 & 5 & 3 & 3 & 3 \\
\hline Parcela 4: La Colorada & 551895 & 866181 & 75 & 6 & 4 & 4 & 4 \\
\hline Parcela 5: San Luis & 553017 & 869702 & 46 & 5 & 5 & 5 & 5 \\
\hline Parcela 6: San Luis & 555129 & 867867 & 90 & 5 & 4 & 4 & 4 \\
\hline Parcela 7: Villa Lourdes & 558713 & 864190 & 78 & 10 & 8 & 8 & 8 \\
\hline Parcela 8: El Ejido & 569538 & 873882 & 51 & 19 & 17 & 17 & 17 \\
\hline Parcela 9: Chupá & 546249 & 859400 & 79 & 5 & 3 & 3 & 3 \\
\hline Total & & & & 70 & 58 & 58 & 58 \\
\hline
\end{tabular}

a La altitud corresponde a metros sobre el nivel del mar (MSNM).

b Número de plantas positivas. Para determinar la especie de begomovirus, se analizaron solamente las muestras que resultaron positivas a begomovirus. El virus del moteado amarillo del tomate (TYMoV) y el virus del enrollamiento de la hoja de tomate de Sinaloa (ToLCSiV) no se identificaron en este estudio. Por tanto, estos virus no se incluyeron en la tabla.

región común $(\mathrm{CR})$ completa y un segmento de la proteína de cubierta $(\mathrm{CP})$, mientras que la segunda pareja de iniciadores amplifica un fragmento de $\sim 500-\mathrm{pb}$, correspondiente a un segmento de la proteína de movimiento (MP) y la región común (CR) completa (Rojas et al., 1993).
La tipificación de begomovirus se realizó mediante PCR, solamente en las muestras que resultaron positivas a begomovirus, siguiendo el protocolo descrito por Herrera-Vásquez et al. (2016). Para ello, se utilizaron iniciadores específicos para las tres especies de begomovirus que infectan 

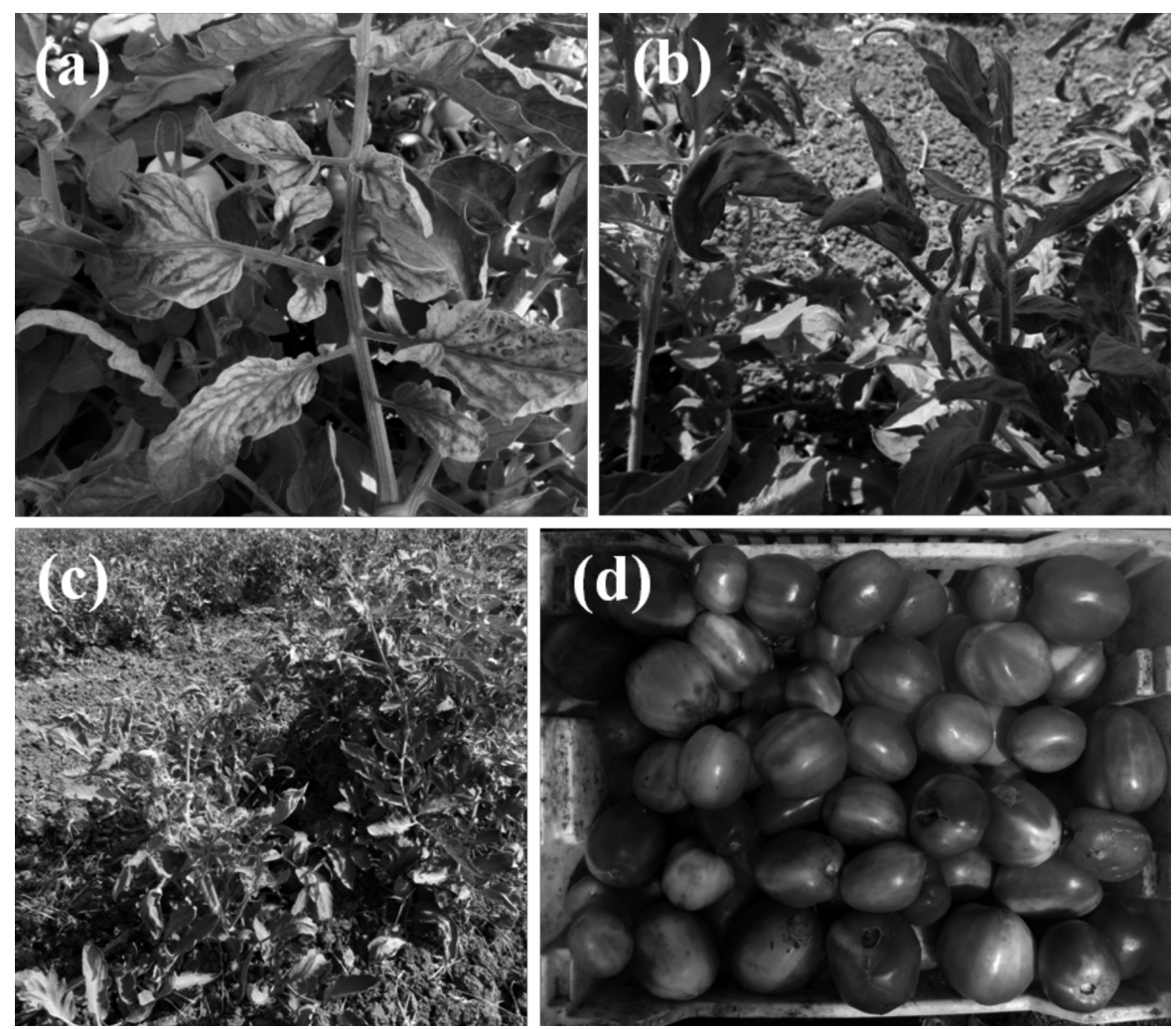

Figura 2. Síntomas de mosaico amarillo de hojas (a); enrollamiento de hojas (b); reducción del tamaño de la planta de tomate (c); maduración irregular y reducción del tamaño de los frutos de tomate (d).

tomate en Panamá (PYMPV, TYMoV y ToLCSiV) (Herrera-Vásquez et al., 2015, 2016). Los iniciadores PYMPVA-151v/PYMPVA-894c (específicos para PYMPV) amplifican un fragmento de 743-pb, correspondiente a un segmento de la CP (HerreraVásquez et al., 2016), mientras que los iniciadores TYMoVClv/TYMoVClc (específicos para TYMoV) y ToLCSiVC2v/ToLCSiVC2c (específicos para ToLCSiV) amplifican un fragmento de 479-pb y 580 -pb, respectivamente, correspondientes a un segmento de la Rep (Nakhla et al., 2005).

Para confirmar la identidad de PYMPV, única especie de begomovirus identificada en este estudio, se secuenciaron dos productos de PCR en el Instituto Smithsonian de Investigaciones Tropicales (STRI) en Panamá. Las secuencias obtenidas se compararon con secuencias de PYMPV disponibles en la base de datos del GenBank, con la ayuda del instrumento de búsqueda de alineamientos locales básicos (BLAST) (Altschul et al., 1997). El árbol de homología se obtuvo de la comparación de las secuencias de PYMPV en el programa DNAMAN versión 4.02 Lynnon Biosoft@1994-98 (Institute of Plant Pathology, BBA, Alemania). Las secuencias de ToLCSiV (AJ608286) y TYMoV (KC176780) se utilizaron como "outgroup". Las secuencias de PYMPV presentadas en este estudio se depositaron en la base de datos del GenBank bajo los números de acceso MF940172 y MF940173.

\section{Resultados y Discusión}

La detección de begomovirus se realizó mediante PCR, utilizando iniciadores degenerados para el ADN-A y el ADN-B de estos virus. Cincuenta y ocho (58) muestras de tomate $(82,9 \%$ del total de muestras recolectadas) (Tabla 1) mostraron el tamaño de banda esperado de $~ 1.400-$ pb y 500-pb para el ADN-A y el ADN-B, respectivamente, tras la electroforesis en gel de agarosa, lo que indica que existe infección por begomovirus bipartito del Nuevo Mundo. No se observó amplificación cuando se utilizó agua ultrapura estéril o plantas sanas de tomate como molde en el análisis de PCR (Figura 3). 
(a) $\begin{array}{lllllllll}1 & 2 & M & 3 & 4 & 5 & 6 & 7 & 8\end{array}$

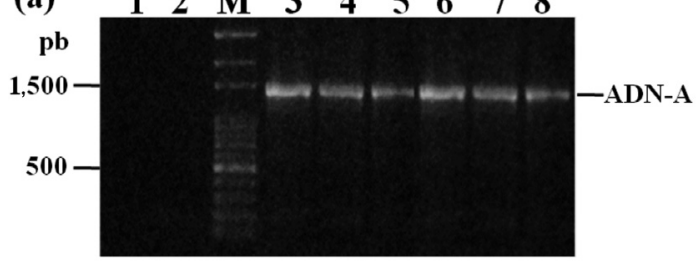

(b) $\begin{array}{lllllllll}1 & 2 & M & 3 & 4 & 5 & 6 & 7 & 8\end{array}$

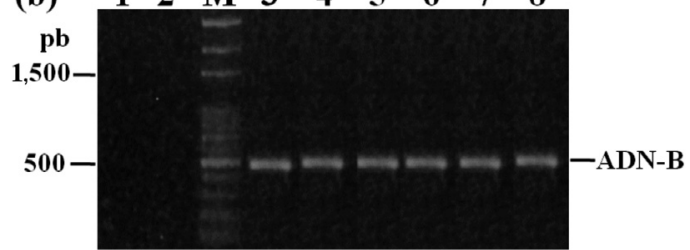

Figura 3. Amplificación mediante PCR de fragmentos de $~ 1.400-$ pb y 500-pb correspondientes al ADN-A (a) y ADN-B (b) de begomovirus, respectivamente. Línea 1, agua ultrapura estéril; Línea 2, planta de tomate sana; Líneas 3-8, muestras de hojas de tomate procedentes de distintas localidades de la provincia de Los Santos; Línea M, marcador de peso molecular de 100-pb (AMRESCO).

La presencia de begomovirus se determinó en todas las parcelas y localidades evaluadas en la provincia de Los Santos, durante el período 20162017 (Tabla 1). La incidencia más alta $(100,0 \%)$ se presentó en las localidades de Tres Quebradas (Parcela 1) y San Luis (Parcela 5), mientras que la incidencia más baja $(60,0 \%)$ se obtuvo en las localidades de Las Cruces (Parcela 3) y Chupá (Parcela 9) (Tabla 1). En estudios previos realizados por Herrera-Vásquez et al. (2016), la incidencia de begomovirus se presentó en el rango de 20-80\% en cultivos de tomate en diferentes localidades de la provincia de Los Santos, incluida Tres Quebradas. No obstante, el presente estudio es el primero que analiza la incidencia de begomovirus en las localidades de Las Cruces, La Colorada, San Luis, Villa Lourdes, El Ejido y Chupá, consideradas las zonas más importantes del cultivo de tomate en esta provincia. Por tanto, la disminución de la producción de tomate industrial reportada recientemente en la provincia de Los Santos, podría estar asociada a la alta incidencia de begomovirus determinada en este estudio, lo que coincide con la presencia en esta región del país de los biotipos A y B de B. tabaci (Alvarado et al., 2004), denominados actualmente grupos Nuevo Mundo (NW) y Oriente Medio Asia Menor 1 (MEAM1), respectivamente (Lee et al., 2013), ambos vectores de begomovirus (Morales, 2006).
La tipificación de begomovirus se realizó mediante PCR en las 58 muestras que mostraron una reacción positiva a este grupo de virus, utilizando iniciadores específicos para PYMPV, TYMoV y ToLCSiV, únicas especies de begomovirus reportadas en tomate en Panamá (Herrera-Vásquez et al., 2015, 2016). Se identificó solamente a PYMPV en el 100,0\% de estas muestras (Tabla 1), coincidiendo con los estudios realizados por Engel et al. (1998) y Herrera-Vásquez et al. (2016), quienes reportan solamente a esta especie infectando cultivos de tomate en la provincia de Los Santos. El producto de PCR amplificado con los iniciadores específicos para este virus, presentó el tamaño de banda esperado de 743-pb tras la electroforesis en gel de agarosa. No se observó amplificación de este fragmento en los controles negativos utilizados como molde en el análisis de PCR (Figura 4).

Un total de 12 muestras de tomate que mostraban síntomas asociados a virus, las que se recolectaron en diferentes localidades de la provincia de Los Santos, resultaron negativas a begomovirus mediante PCR (Tabla 1). Estas muestras podrían albergar la presencia de otros virus que infectan tomate en distintas regiones de Panamá, como el virus Y de la papa (PVY; género Potyvirus, familia Potyviridae) (Herrera-Vásquez et al., 2009b), virus del mosaico del pepino (CMV; género Cucumovirus, familia Bromoviridae), virus del torrado del tomate (ToTV; género Torradovirus, familia Secoviridae) (HerreraVasquez et al., 2009a), virus del mosaico del tabaco (TMV) y virus del mosaico del tomate (ToMV), ambos pertenecientes al género Tobamovirus dentro de la familia Virgaviridae (J.A. Herrera-Vásquez,

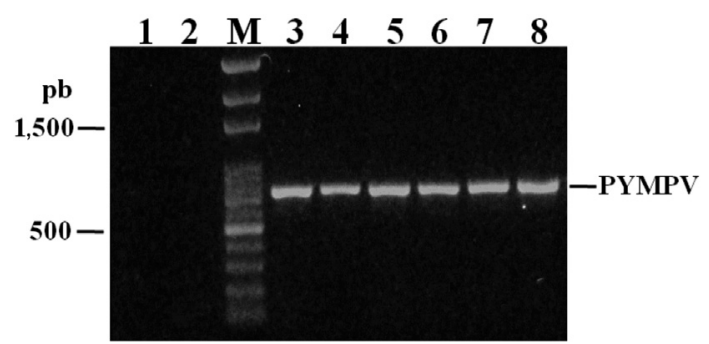

Figura 4. Amplificación mediante PCR de un fragmento de 743-pb correspondiente a la proteína de cubierta (CP) del virus del mosaico amarillo de la papa de Panamá (PYMPV). Línea 1, agua ultrapura estéril; Línea 2, planta de tomate sana; Líneas 3-8, muestras de hojas de tomate procedentes de distintas localidades de la provincia de Los Santos; Línea M, marcador de peso molecular de 100-pb (AMRESCO). 
comunicación personal). PVY y CMV se transmiten por áfidos de forma no persistente (Brunt et al., 1996), mientras que ToTV se transmite por las moscas blancas B. tabaci, Trialeurodes vaporariorum Westwood y Trialeurodes abutilonea Haldeman de forma semipersistente (Verbeek et al., 2014). En Panamá, se reporta la presencia solamente de $B$. tabaci y $T$. vaporariorum (Zachrisson y Poveda, 1992). TMV y ToMV se transmiten mecánicamente y por semilla (Brunt et al., 1996). La presencia o ausencia de estos virus en las muestras que resultaron negativas a begomovirus podría analizarse en un futuro estudio.

Las secuencias de PYMPV obtenidas en este estudio (MF940172 y MF940173), quienes se compararon con las secuencias de este virus obtenidas en 1998 (Y15034), 2011 (KP313716) y 2012 (KP313717), mostraron una alta homología (97-100\%) a nivel de secuencia de nucleótidos en la zona del genoma estudiada (CP) (Figura 5). Por tanto, estos resultados aportan evidencia de que PYMPV se ha diseminado durante los últimos 20 años en Panamá con una variabilidad genética muy baja, lo que es consistente con los trabajos realizados para virus de plantas, que indican que la estabilidad genética en lugar de la diversidad podría ser la regla general de este grupo de virus, independientemente si su genoma es ARN o ADN (Gibbs et al., 1999).

\section{Conclusiones}

Las prospecciones realizadas históricamente en cultivos de tomate de la provincia de Los Santos, revelan que las infecciones causadas por PYMPV son de especial preocupación en esta región del país. Esta información podría ayudar a establecer una base para el diseño de estrategias de control de las epidemias causadas por begomovirus en los cultivos de tomate panameños.

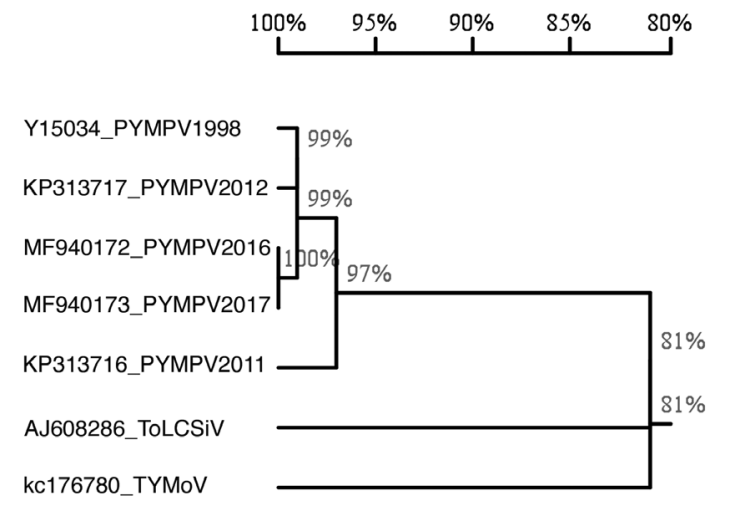

Figura 5. Árbol de homología de la secuencia parcial de la proteína de cubierta (CP) obtenido con el programa DNAMAN. En el análisis realizado se incluyeron las secuencias del virus del mosaico amarillo de la papa de Panamá (PYMPV) obtenidas en este estudio en 2016 (MF940172) y 2017 (MF940173), las que se compararon con secuencias de este virus obtenidas en 1998 (Y15034), 2011 (KP313716) y 2012 (KP313717). Las secuencias del virus del enrollamiento de la hoja de tomate de Sinaloa (ToLCSiV) (AJ608286) y virus del moteado amarillo del tomate (TYMoV) (KC176780) se utilizaron como "outgroup".

\section{Agradecimientos}

Los autores expresan su agradecimiento a la Secretaría Nacional de Ciencia, Tecnología e Innovación (SENACYT, Panamá) por el financiamiento de esta investigación, por medio del proyecto FID14-020. El Dr. J.A. HerreraVázquez fue apoyado por el Sistema Nacional de Investigación (SNI, Panamá). Agradecemos a I. Zachrisson (CIAC, IDIAP, Panamá) por su asistencia técnica en el mapeo de las coordenadas UTM. Finalmente, queremos agradecer al personal técnico de la Dirección Nacional de Sanidad Vegetal del Ministerio de Desarrollo Agropecuario (DNSV, MIDA, Panamá) y del IDIAP, así como a los productores de tomate de la provincia de Los Santos por su apoyo y hospitalidad.

\section{Literatura Citada}

Altschul, S.F.; Madden, T.L.; Schaffer, A.A.; Zhang, J.; Zhang, Z.; Miller, W. y Lipman, D.J.

1997. Gapped BLAST and PSIBLAST: a new generation of protein database search programs. Nucleic Acids Research, 25 (17): 3389-3402.

Alvarado, L.; Sánchez, J.; Zachrisson, B. y Fernández, O. 2004. Distribución del biotipo B de Bemisia tabaci en la zona central de Panamá. Manejo Integrado Plagas y Agroecología, 71: 67-72.
Brunt, A.A.; Crabtree, K.; Dallwitz, M.J; Gibbs, A.J.; Watson, L. y Zurcher, E.J. (eds).

1996. Plant Viruses Online: Descriptions and Lists from the VIDE Database. Version: 16 Jh January 1997. Disponible en: http://biology.anu. edu.au/Groups/MES/vide/. Consultado: 18/sep/2017.

Dellaporta, S.; Woods, H. y Hicks, J.

1983. A plant DNA minipreparation: version II. Plant Molecular Biology Reporter, 1 (4): 19-21. 
Engel, M.; Fernández, O.; Jeske, H. y Frischmuth, T. 1998. Molecular characterization of a new whiteflytransmissible bipartite geminivirus infecting tomato in Panama. Journal of General Virology, 79: 2313-2317.

\section{FAO}

2017. Base de datos estadísticos FAOSTAT. Disponible en: http://faostat3.fao.org/faostat-gateway/go/to/download/Q/ QC/E. Consultado: 27/jul/2017.

Gibbs, A.J.; Keese, P.L.; Gibbs, M.J. y García-Arenal, F. 1999. Plant virus evolution: Past, present and future. In: Domingo, E.; Webster, R.; Holland, J. Origin and Evolution of Viruses, pp. 263-285.

Hanssen, I.M.; Lapidot, M. y Thomma, B.P.H.J.

2010. Emerging viral diseases of tomato crops. Molecular Plant-Microbe Interactions, 23 (5): 539-548.

Herrera Vásquez, J.A.; Ortega, D.; Romero, A.B.; Davino, S.;

Mejía, L.C.; Panno, S. y Davino, M.

2015. First report of Tomato leaf curl Sinaloa virus infecting tomato crops in Panama. New Disease Reports, 31: 30.

Herrera Vásquez, J.A.; Ortega, D.; Romero, A.B.; Davino, S.;

Mejía, L.C.; Panno, S. y Davino, M.

2016. Begomoviruses infecting tomato crops in Panama. Journal of Phytopathology, 164 (2): 102-113.

Herrera Vásquez, J.A.; Alfaro-Fernández, A.; Córdoba-Sellés, M.C.; Cebrián, M.C.; Font, M.I. y Jordá, C.

2009a. First report of Tomato torrado virus infecting tomato in single and mixed infections with Cucumber mosaic virus in Panama. Plant Disease, 93: 198.

Herrera Vásquez, J.A.; Córdoba-Sellés, M.C.; Cebrián, M.C.;

Alfaro-Fernández, A y Jordá, C.

2009b. First report of Pepper mild mottle virus and Tobacco mild green mosaic virus infecting pepper in Panama. Plant INEC Pathology, 58: 786

2017. Volumen IV. Cultivos Anuales o Temporales, Cuadro $\mathrm{N}^{\circ} 18$. Disponible en: https://www.contraloria. gob.pa/inec/Publicaciones/Publicaciones.aspx?ID_
SUBCATEGORIA $=60 \&$ ID_PUBLICACION $=479 \&$ ID IDIOMA=1\&ID_CATEGORIA=15. Consultado el 27/07/2017

Jones, D.R.

2003. Plant viruses transmitted by whiteflies. European Journal of Plant Pathology, 109 (3): 195-219.

Lee, W.; Park, J.; Lee, G.S.; Lee, S. y Akimoto, S.I.

2013. Taxonomic status of the Bemisia tabaci complex (Hemiptera: Aleyrodidae) and reassessment of the number of its constituent species. PLoS ONE, 8:e63817. doi:10.1371/ journal.pone.0063817.

Mendoza, G.

2015. Periódico El Siglo, Panamá. Disponible en: http:// elsiglo.com.pa/economia/tomateros-pierden-50produccion/23841577. Consultado: 09/ago/2017.

Morales, F.J.

2006. History and current distribution of begomoviruses in Latin America. Advances in Virus Research, 67: 127-162.

Nakhla, M.; Sorenson, A.; Mejía, L.; Ramírez, P.; Karkashian,

J. y Maxwell, D.

2005. Molecular characterization of tomato-infecting begomoviruses in Central America and development of DNA-based detection methods. Acta Horticulturae, 695: 277-288.

Rojas, M.R.; Gilbertson, R.L.; Russell, D.R. y Maxwell, D.P. 1993. Use of degenerate primers in the polymerase chain reaction to detect whitefly-transmitted geminiviruses. Plant Disease, 77: 340-347.

Verbeek, M.; Van Bekkum, P.J.; Dullemans, A.M. y Van der Vlugt, R.A.

2014. Torradoviruses are transmitted in a semipersistent and stylet-borne manner by three whitefly vectors. Virus Research, 186: 55-60.

Zachrisson, B. y Poveda, J.

1992. Las moscas blancas en Panamá. In: Hilje, L., Arboleada, O. (eds.). Las moscas blancas (Homoptera: Aleyrodidae) en América Central y el Caribe. Turrialba, CR, CATIE, pp. 64-66. 
